\title{
Characterization and predicted role of microRNA expression profiles associated with early childhood obesity
}

\author{
SHENGRONG OUYANG ${ }^{1}$, RENQIAO TANG ${ }^{2}$, ZHUO LIU $^{1}$, FEIFEI MA ${ }^{1}$, YUANYUAN LI $^{1}$ and JIANXIN WU ${ }^{1}$ \\ ${ }^{1}$ Department of Biochemistry, Capital Institute of Pediatrics, Beijing 100020; \\ ${ }^{2}$ Graduate School of Peking Union Medical College, Beijing 100730, P.R. China
}

Received August 10, 2016; Accepted May 8, 2017

DOI: $10.3892 / \mathrm{mmr} .2017 .7118$

\begin{abstract}
MicroRNAs (miRNAs) are implicated in the pathogenesis of obesity. The aim of the present study was to characterize the miRNA profile associated with early childhood obesity in peripheral blood mononuclear cells (PBMCs). A total of 12 children ( 6 obese and 6 lean controls) aged 36 months old to 48 months old were recruited. The miRNA expression profile from PBMCs was detected using the multiplexed NanoString nCounter system. Bioinformatics was employed to detect target genes and miRNA-regulated biological function. A total of 9 differentially expressed miRNAs were identified in obese children compared with lean children $(\mathrm{P}<0.05)$. Among the 9 miRNAs, miR-199a-3p/miR-199b-3p and miR-4454 presented at least a 1.5-fold change in expression. A total of 643 potential target genes were regulated by the three miRNAs, and 291 of the potential genes were involved in a protein interaction network. Gene ontology annotation indicated that 291 potential genes were enriched in 14 biological process annotations and 2 molecular function annotations. miRNA dysregulation may be involved in early childhood obesity.
\end{abstract}

\section{Introduction}

Obesity is a major public health problem worldwide. Childhood obesity increases the risk of obesity in adulthood, which is associated with the development of obesity-related disorders,

Correspondence to: Professor Jianxin Wu, Department of Biochemistry, Capital Institute of Pediatrics, 2 Yabao Road, Beijing 100020, P.R. China

E-mail: cipbiolab@163.com

Abbreviations: MiRNA, microRNA; PBMC, peripheral blood mononuclear cell; qPCR, quantitative polymerase chain reaction; BMI, body mass index; STRING, Search Tool for the Retrieval of Interacting Genes; PPI, protein-protein interaction; DAVID, Database for Annotation, Visualization and Integration Discovery; GO, gene ontology

Key words: obesity, microRNA, preschool children, peripheral blood mononuclear cell, expression profile such as hypertension, hyperlipidemia, insulin resistance and diabetes mellitus.

MicroRNAs (miRNAs) are highly conserved non-coding RNA molecules of 22 nucleotides that exert post-transcriptional effects on gene expression. These RNA molecules generally bind to a target sequence localized in the 3'-untranslated region of their target messenger RNAs and regulate protein translation or mRNA stability $(1,2)$. miRNAs are involved in highly regulated processes, such as proliferation, differentiation, apoptosis and metabolic processes. Dysregulation of miRNA expression is closely associated with many diseases, including obesity (3-6). However, only a few studies, such as that of Hulsmans et al (7) have focused on the relationship between obesity and miRNAs in peripheral blood mononuclear cells (PBMCs) (7).

Early childhood provides an excellent opportunity to investigate the occurrence and development of obesity without confounding lifestyle habits (e.g., smoking) and co-existing inflammatory conditions (e.g., cardiovascular disease and arthritis) (8). In the present study, the authors characterized the miRNA profile in PBMCs of obese children aged 36 months old to 48 months old using a multiplexed NanoString nCounter system. NanoString nCounter is more sensitive than microarray, and has similar sensitivity to quantitative polymerase chain reaction (qPCR) (9). Thus, this approach is used to profile miRNAs in PBMCs associated with early childhood obesity.

\section{Materials and methods}

Study population. Subjects were recruited from the children (aged 36 months old to 48 months old) who participated in a physical examination for kindergarten enrollment (10). Children with underlying hormone deficiencies, genetic disorders, inflammatory conditions, or occurrences of recent acute infections were excluded. A total of 6 obese children [body mass index $(\mathrm{BMI})>18.5$ and $<26 \mathrm{~kg} / \mathrm{m}^{2}$ ] and six lean controls (BMI $>13.5$ and $<15 \mathrm{~kg} / \mathrm{m}^{2}$ ) were randomly selected for a miRNA expression profile analysis. Obesity classification criteria refer to Chinese children aged 3 years old with a BMI measurement of $28 \mathrm{~kg} / \mathrm{m}^{2}\left(\mathrm{BMI}_{28}\right)(11)$. The study was approved by the Institutional Ethics Committee of the Capital Institute of Pediatrics (Beijing, China).

Isolation of PBMCs and RNA extraction. Blood samples were collected, and PBMCs were isolated by gradient separation 
using Histopaque-1077 (Sigma-Aldrich; Merck KGaA; Darmstadt, Germany) following removal of the plasma fraction. Total RNA was isolated using the mirRNeasy Mini kit (Qiagen GmbH, Hilden, Germany) following the manufacturer's protocol. Total RNA concentration and RNA ratios (260/230 and 260/280) were determined with a NanoDrop 2000 spectrophotometer (Thermo Fisher Scientific, Inc., Waltham, MA, USA).

NanoString $n$ Counter miRNA assay for miRNA profiling. The multiplexed NanoString nCounter miRNA expression assay (Human miRNA Assay 2.0 kit) was employed for profiling 800 human miRNAs (NanoString Technologies, Inc., Seattle, WA, USA). The assay was performed according to manufacturer's protocol. In brief, the NanoString nCounter platform involved mixing total RNA with pairs of capture and reporter probes tailored to each miRNA, hybridizing, washing away excess probes, immobilizing probe-bound miRNAs on a surface, and scanning color-coded bar tags on the reporter probes. Total RNA (100 ng) was used as input material, with $3 \mu 1$ total volume for each sample. All hybridization reactions were incubated at $65^{\circ} \mathrm{C}$ for $18 \mathrm{~h}$, and a max-density scan (555 fields of view) was selected.

Preprocessing of miRNA panel codeset. The nCounter assay for each sample consisted of six positive controls, eight negative controls, five control mRNAs (ACTB, B2M, GAPDH, RPL19 and RPLP0) and 800 miRNAs. Each sample was normalized according to the geometric mean of the top 100 most highly expressed miRNAs. The mean plus twice the standard deviation of the eight negative controls for each sample was subtracted from each miRNA count in that sample. Only miRNAs with non-negative counts across all samples were retained for downstream analysis.

Bioinformatics methods to target gene prediction. The target gene sets for miRNAs (i.e., miR-199a-3p, miR-199b-3p and miR-4454) were determined using TarBase v7.0 (http://www.microrna.gr/tarbase). TarBase v7.0 provides hundreds of thousands of high-quality manually curated experimentally validated miRNA: Gene interactions enhanced with detailed metadata (12). The protein-protein interaction (PPI) network was constructed for the target genes using information provided by the Search Tool for the Retrieval of Interacting Genes (STRING; string-db.org) (13) and was subsequently visualized with Cytoscape 3.2.1 (14). Interactions with the highest confidence (score $\geq 0.9$ ) were retained in the network. Proteins in the network served as the 'nodes,' and each pairwise protein interaction (referred to as an 'edge') was presented as an undirected link. Gene degrees were calculated and genes with $>10$ degrees in the PPI network were considered hub genes. The Database for Annotation, Visualization and Integration Discovery (DAVID; david.abcc.ncifcrf. gov) (15) was applied to perform gene ontology (GO) enrichment analyses for the predicted miRNA target genes. $\mathrm{P}<0.05$ and false discovery rate $<0.05$ were selected as the threshold. The miRNA-GO network is built according to the relationship of miRNAs and significant GOs. In the miRNA-GO network, a circle represents a GO term and a red square represents miRNA, and their relationship is represented by one edge.
The center of the network is represented by degree. Degree of miRNA means the number of links one miRNA regulates GOs.

Statistical analysis. All variables were expressed as mean and standard deviation. Student's t-test was applied to compare the basic characteristics (i.e., age and BMI) and identify differentially expressed miRNAs between groups (i.e., obese vs. lean). The miRNA code counts were $\log 2$-transformed prior to Student's t-test. Microsoft Excel 10.0 (Microsoft Corporation, Redmond, WA, USA) was employed for all statistical analyses, and $\mathrm{P}<0.05$ was used to indicate a statistically significant difference. The data process for the NanoString nCounter was performed using nSolver 2.0 (NanoString Technologies, Inc.).

\section{Results}

Basic characteristics of the studied children. Table I lists the basic characteristics of the recruited children. Statistically significant differences were not observed between the two groups (i.e., obese vs. lean) in terms of age and gender, whereas the difference in BMI was significant as expected $(\mathrm{P}<0.001)$.

\section{Differentially expressed miRNAs in the NanoString $n$ Counter assay. A total of 155 of the 800 miRNAs profiled using the NanoString nCounter assay were detected above background across all samples. miRNAs $>100$ code counts were retained for downstream analysis. The dataset (i.e., 76 miRNAs for 12 samples) was retained and underwent $\log 2$ transformation prior to statistical analysis (Fig. 1). A total of nine differen- tially expressed miRNAs were identified in obese children relative to lean children $(\mathrm{P}<0.05$; Table I and Fig. 2). Among these miRNAs, miR-199a-3p/miR-199b-3p and miR-4454 had at least a 1.5-fold change in expression (Fig. 2A).}

Protein interaction network prediction and GO analysis. The authors restricted the protein interaction network and GO analyses to the genes that were predicted as targets of the three miRNAs (i.e., miR-199a-3p, -199b-3p, and -4454). A total of 643 target genes potentially regulated by the three miRNAs were searched from the TarBase database. From this data set, a PPI network that contains 291 genes was constructed using the STRING platform following excluding the isolated nodes (Fig. 3). The degree was then calculated for each node in the network to screen for hub genes, which are generally considered to serve a critical role in disease development. A total of 14 hub genes with a degree $>10$ were identified and filled with red in the PPI network (Table II and Fig. 3). To gain a high-level view of gene functions that may be affected by the altered miRNA expression in obesity, the 291 genes were mapped to the GO categories. A total of 14 annotations within the biological process and 2 annotations within the molecular function were enriched (Table III). To identify which miRNAs serve a critical role in biological process or molecular function, miRNA-GO network was analyzed by bioinformatics analysis. As demonstrated in Fig. 4, hsa-miR-199a-3p had the highest degree, thereby indicating that the miRNA was involved in more biological functions than others. 
Table I. Characteristics of the participants and differentially expressed miRNAs.

\begin{tabular}{|c|c|c|c|c|}
\hline Characteristic & Lean $(n=6)$ & Obese $(n=6)$ & P-value ${ }^{a}$ & Fold-change \\
\hline Age (month), mean \pm SD & $39.2 \pm 2.3$ & $39.7 \pm 2.2$ & 0.71 & - \\
\hline Gender (male/female) & $3 / 3$ & $3 / 3$ & - & - \\
\hline BMI $(\mathrm{kg} / \mathrm{m} 2)$, mean $\pm \mathrm{SD}$ & $14.1 \pm 0.5$ & $23.2 \pm 1.5$ & $<0.001$ & - \\
\hline $\operatorname{miR}-301 a-3 p$ & $6.71 \pm 0.29$ & $7.13 \pm 0.16$ & 0.011 & 1.33 \\
\hline miR-199a-3p/-199b-3p & $10.44 \pm 0.39$ & $11.03 \pm 0.32$ & 0.017 & 1.5 \\
\hline miR-191-5p & $9.87 \pm 0.19$ & $10.21 \pm 0.22$ & 0.018 & 1.26 \\
\hline miR-130a-3p & $8.45 \pm 0.29$ & $8.92 \pm 0.29$ & 0.018 & 1.38 \\
\hline miR-361-5p & $6.18 \pm 0.18$ & $6.6 \pm 0.36$ & 0.031 & 1.33 \\
\hline miR-126-3p & $8.24 \pm 0.26$ & $8.58 \pm 0.24$ & 0.042 & 1.26 \\
\hline let-7g-5p & $12.29 \pm 0.16$ & $12.07 \pm 0.17$ & 0.043 & -1.16 \\
\hline $\operatorname{miR}-4454$ & $9.97 \pm 1.13$ & $8.88 \pm 0.2$ & 0.043 & -2.12 \\
\hline
\end{tabular}

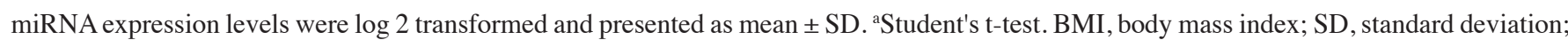
miRNA, microRNA.

\section{Discussion}

Obesity is associated with a chronic low-grade inflammatory state. Monocyte migration and subsequent transformation into macrophages within diseased tissues are key factors in self-perpetuating inflammation associated with metabolic disorders. Increased macrophage populations during obesity result from an influx of circulating monocytes. Ghanim et al (16) were the first to show that PBMCs in obesity are in a proinflammatory state with increased intranuclear nuclear factor $-\kappa \mathrm{B}$ binding and decreased I $\mathrm{B}-\beta$. An increasing amount of evidence highlights that miRNAs can be important in the immune/inflammatory system $(17,18)$. The present study identified 155 miRNAs that were especially expressed in PBMCs of children aged 36 months old to 48 months old. The present study is the first to link the altered expression of miRNAs to PBMCs from obese preschool children.

The role of miRNAs in adipogenesis, adipocyte differentiation, fat metabolism and insulin secretion and action during obesity has been increasingly recognized (19). Peng et al (20) reviewed adipogenic miRNAs (e.g., miR-143, -103, -200, -30, $-204 / 211,-375$ and $-519 d$ ) and antiadipogenic miRNAs (e.g., miR-145, -155 and -448). The majority of studies focused on adipose tissue, adipocyte, plasma and serum, but only a few focused on PBMCs, in terms of the effect of miRNA on human obesity. Among the miRNAs expressed in PBMCs, miR-33a, $-33 b,-935$ and -4772 were upregulated (4,5), whereas miR-223, -224, -376b, -181a and -146b-5p were downregulated in obese people as compared with normal controls $(3,5,7)$. Two studies investigated the relationship between miRNAs and obese children. Carolan et al (4) determined a 4-fold increase in miR-33a expression $(\mathrm{P}=0.001)$ and a 3 -fold increase in miR-33b expression $(\mathrm{P}=0.017)$ in the PBMCs of obese children. Prats-Puig et al (6) indicated that 15 specific circulating miRNAs were significantly dysregulated in prepubertal obesity, including decreased miR-221 and miR-28-3p concentrations and increased plasma concentrations of miR-486-5p, -486-3p, -142-3p, -130b and -423-5p (all $\mathrm{P}<0.0001$ ). However,
Table II. A total of 14 hub genes with a degree of $>10$ in the protein-protein interaction network.

\begin{tabular}{lc}
\hline Symbol & Degree \\
\hline CDC42 & 26 \\
MAPK1 & 18 \\
MAPK8 & 17 \\
CDKN1A & 16 \\
CREB1 & 14 \\
PIK3R1 & 14 \\
FOS & 13 \\
MAPRE1 & 12 \\
PSMA6 & 12 \\
ARHGEF12 & 12 \\
PSMD6 & 12 \\
NUP43 & 12 \\
YWHAZ & 11 \\
MTOR & 11 \\
\hline
\end{tabular}

specifically expressed miRNAs associated with early childhood obesity have not been reported. The current study identified nine differentially expressed miRNAs $(\mathrm{P}<0.05)$, where miR-199a, -199b-3p and -4454 were altered by at least 1.5-fold in preschool children compared with normal controls. Therefore, the authors selected these three miRNAs for further bioinformatics analysis.

Hsa-miR-199a-3p/-199b-3p was initially cloned by Landgraf et al (21). Subsequently, miR-199a-3p was deeply sequenced from human mesenchymal stem cells by Koh et al (22). Several studies recently reported the role of miR-199a-3p in obesity. Nesca et al (23) revealed that a specific rise in the levels of several miRNAs, including miR-199a-3p, primarily occur in obesity-associated diabetic mice and result in increased beta cell apoptosis. Shi et al (24) identified that miR-199a-3p levels were increased in differentiating 


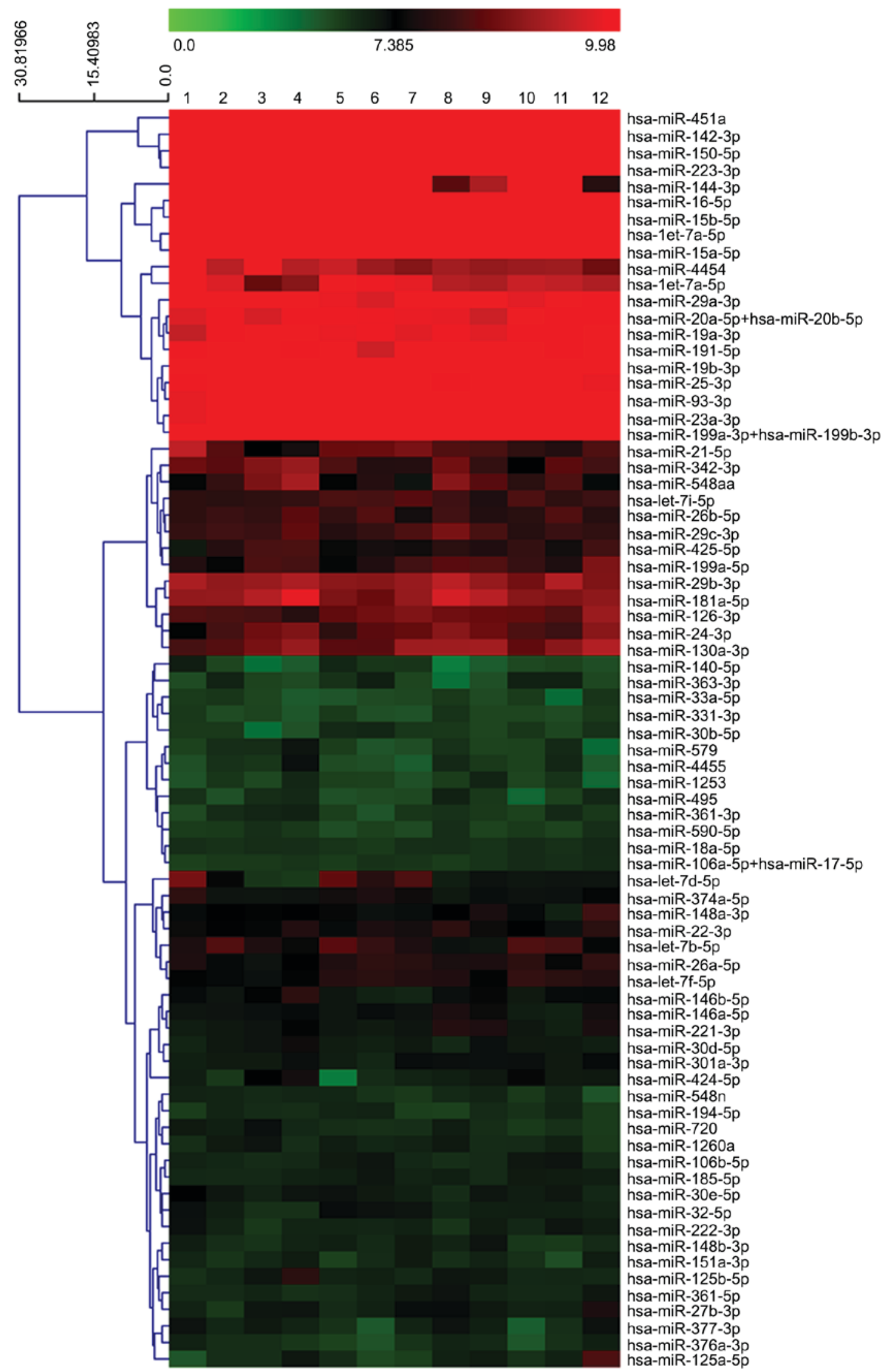

Figure 1. Analysis of differentially-expressed miRNAs. A total of 76 miRNAs with an expression level greater than 100 code counts are presented. 1-6, lean samples; 7-12, obese samples; miRNA, microRNA.

human adipose-derived mesenchymal stem cells. The present study also determined that miR-199a-3p was upregulated in obese children compared with lean controls. However, Ortega et al (25) demonstrated that surgery-induced weight 
Table III. GO enrichment analysis of biological process and molecular function.

\begin{tabular}{|c|c|c|c|c|}
\hline GO term & GO name & Count & P-value & FDR \\
\hline \multicolumn{5}{|c|}{ Biological progress } \\
\hline GO:0007049 & Cell cycle & 40 & $2.47 \mathrm{E}-08$ & 4.24E-05 \\
\hline GO:0033043 & Regulation of organelle organization & 18 & $8.86 \mathrm{E}-07$ & 0.0015 \\
\hline GO:0010604 & Positive regulation of macromolecule metabolic process & 39 & $9.30 \mathrm{E}-07$ & 0.0015 \\
\hline GO:0000278 & Mitotic cell cycle & 23 & $2.44 \mathrm{E}-06$ & 0.0041 \\
\hline GO:0031328 & Positive regulation of cellular biosynthetic process & 32 & $6.92 \mathrm{E}-06$ & 0.0118 \\
\hline GO:0016310 & Phosphorylation & 35 & $9.19 \mathrm{E}-06$ & 0.0157 \\
\hline GO:0009891 & Positive regulation of biosynthetic process & 32 & $9.24 \mathrm{E}-06$ & 0.0158 \\
\hline GO:0022402 & Cell cycle process & 28 & $1.07 \mathrm{E}-05$ & 0.0183 \\
\hline GO:0051301 & Cell division & 19 & $1.46 \mathrm{E}-05$ & 0.0250 \\
\hline GO:0051173 & Positive regulation of nitrogen compound metabolic process & 30 & $1.54 \mathrm{E}-05$ & 0.0264 \\
\hline GO:0006793 & Phosphorus metabolic process & 39 & $1.81 \mathrm{E}-05$ & 0.0311 \\
\hline GO:0006796 & Phosphate metabolic process & 39 & $1.81 \mathrm{E}-05$ & 0.0311 \\
\hline GO:0010557 & Positive regulation of macromolecule biosynthetic process & 30 & $2.05 \mathrm{E}-05$ & 0.0351 \\
\hline GO:0045935 & $\begin{array}{l}\text { Positive regulation of nucleobase, nucleoside, nucleotide } \\
\text { and nucleic acid metabolic process }\end{array}$ & 29 & $2.32 \mathrm{E}-05$ & 0.0397 \\
\hline \multicolumn{5}{|c|}{ Molecular function } \\
\hline GO:0008134 & Transcription factor binding & 30 & $1.77 \mathrm{E}-07$ & 2.58E-04 \\
\hline GO:0004674 & Protein serine/threonine kinase activity & 23 & $2.81 \mathrm{E}-05$ & 0.04088 \\
\hline
\end{tabular}

GO, gene ontology; FDR, false discovery rate.
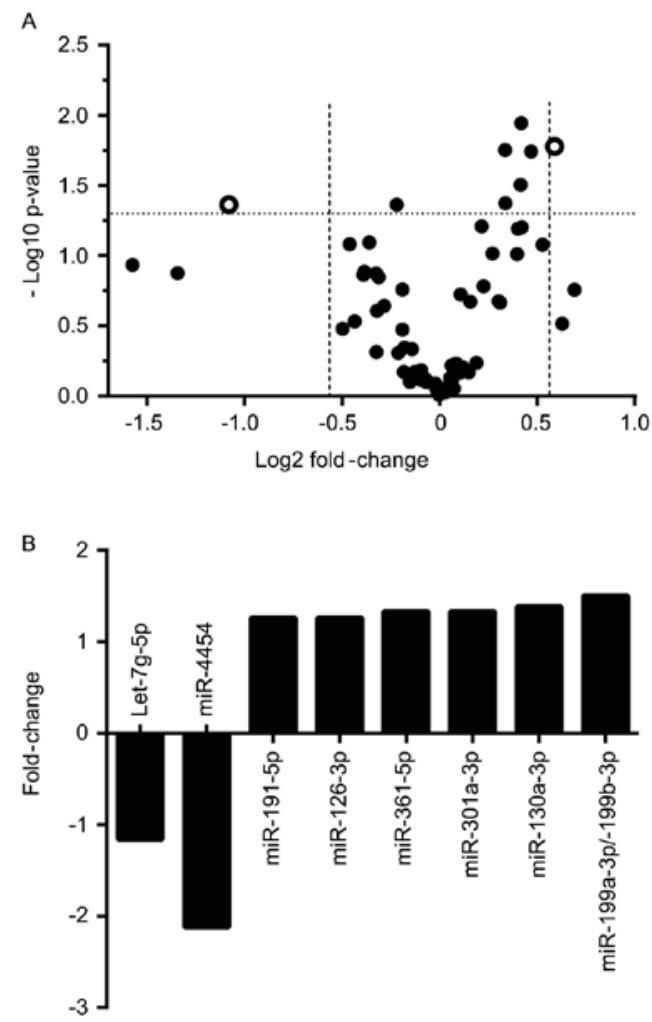

Figure 2. Identification of miRNAs that were differentially expressed between groups (obese vs. lean). Among these miRNAs, miR-199a-3p/miR-199b-3p and miR-4454 exhibited at least a 1.5-fold change in expression. (A) Volcano plot of statistical significance against fold-change of differentially-expressed miRNAs. $\mathrm{P}<0.05$ was considered to indicate a statistically-significant difference. (B) Fold-change of differentially-expressed miRNAs. miRNA, microRNA. loss leads to significant upregulation of miR-199a-3p in the plasma, which conflicts with the authors' finding and that of two other studies $(23,24)$. Hsa-mir-4454 was initially identified by deep sequencing in human B cells (26). However, no study has explored the relationship between obesity and miR-4454, as well as miR-199b-3p. Thus, the biological effects of miR-199b-3p and miR-4454 on obesity must be further clarified. In addition, the reported functions of the three miRNAs include involvement in other diseases or physiological process, such as type 2 diabetes (miR-199a-3p) (27), lipid regulation (miR-199a-3p) (28), chondrogenesis (miR-199b-3p) (29), hepatocellular cancer (miR-199a/-199b-3p) (30-32) and colon cancer (miR-4454) (33).

Intricate molecular networks mediated by miRNAs can form a robust mechanism to generate rapid and potent responses to cellular events during obesity occurrence and development. Weisberg et al (34) reported that obesity pathogenesis involves many regulatory pathways, including transcriptional regulatory networks. The result of the PPI network construction shows that many proteins are linked; 14 genes are hub genes in the network (Fig. 2). Hub genes have high probabilities of engaging in essential biological functions $(35,36)$. In the present study, hub genes such as CDC42 (37), MAPK1 (38) and MAPK8 (39) have been reported for their roles in adipogenesis and obesity. In addition, the target genes of the three miRNAs were demonstrated to serve a role in some processes, including cell cycle, regulation of organelle organization, positive regulation of macromolecule metabolic process, mitotic cell cycle and positive regulation of nitrogen compound metabolic process. The three miRNAs were associated with the target genes involved in transcription factor binding and 


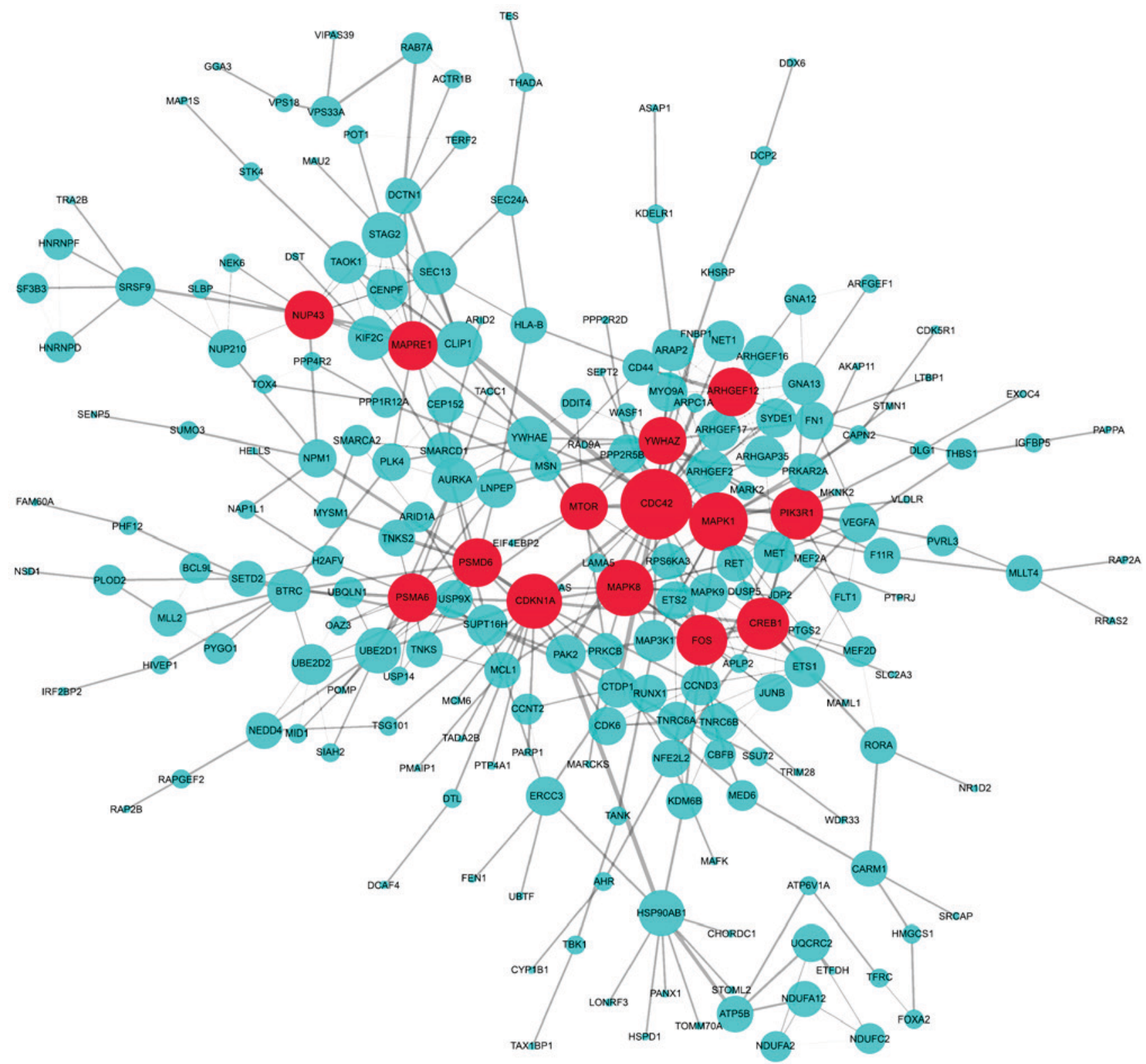

Figure 3. Protein-protein interaction network of predicted miRNA-targeted genes. The 14 hub genes with a degree of $>10$ are indicated in red. The size of the node indicates the number of interactions with other nodes within the network. miRNA, microRNA.



Figure 4. miRNA-GO network analysis. Red box nodes represent miRNAs, and green circle nodes represent GO terms. Edges indicate the inhibitory effect of miRNAs on target genes. GO, gene ontology; miRNA, microRNA. 
protein serine/threonine kinase activity. Of the three miRNAs, miR-199a-3p was involved in more biological functions than the others; thereby indicating that miR-199a-3p possibly plays a more important role in early childhood obesity. Thus, miRNAs can participate in obesity pathogenesis by fine-tuning target gene expression.

However, several limitations of the present study should be considered. First, the sample size should be increased because only 12 samples (6 obese vs. 6 lean) were included in the present study. Secondly, although a NanoString nCounter system is more sensitive than microarray and has similar sensitivity to qPCR (9), the differentially expressed miRNAs should be further cross-validated using qPCR.

The current study characterized a specific miRNA expression profile in PBMCs of obese children aged 36 to 48 months. However, although the functions of some miRNAs are not yet fully understood, miRNAs have emerged as key regulators of gene expression. Results suggested that miRNAs are specifically involved in early childhood obesity. In addition, the authors provided a target gene map of miRNAs that can contribute to future investigations on the regulatory mechanisms of miRNA in childhood obesity. Nevertheless, further investigations are necessary to validate the differential expression of miRNAs in a large sample size and to understand the potential role of individual miRNA in early childhood obesity.

\section{Acknowledgments}

The present study was supported by the National Basic Research Program of China '973' (grant no. 2013CB530605).

\section{References}

1. Djuranovic S, Nahvi A and Green R: A parsimonious model for gene regulation by miRNAs. Science 331: 550-553, 2011.

2. Bartel DP: MicroRNAs: Genomics, biogenesis, mechanism, and function. Cell 116: 281-297, 2004.

3. Hulsmans M, Van Dooren E, Mathieu C and Holvoet P: Decrease of miR-146b-5p in monocytes during obesity is associated with loss of the anti-inflammatory but not insulin signaling action of adiponectin. PLoS One 7: e32794, 2012.

4. Carolan E, Hogan AE, Corrigan M, Gaotswe G, O'Connell J, Foley N, O'Neill LA, Cody D and O'Shea D: The impact of childhood obesity on inflammation, innate immune cell frequency, and metabolic microRNA expression. J Clin Endocrinol Metab 99: E474-E478, 2014.

5. Milagro FI, Miranda J, Portillo MP, Fernandez-Quintela A, Campión J and Martínez JA: High-throughput sequencing of microRNAs in peripheral blood mononuclear cells: Identification of potential weight loss biomarkers. PLoS One 8: e54319, 2013.

6. Prats-Puig A, Ortega FJ, Mercader JM, Moreno-Navarrete JM, Moreno M, Bonet N, Ricart W, López-Bermejo A and Fernández-Real JM: Changes in circulating microRNAs are associated with childhood obesity. J Clin Endocrinol Metab 98 : E1655-E1660, 2013.

7. Hulsmans M, Sinnaeve P, Van der Schueren B, Mathieu C, Janssens $\mathrm{S}$ and Holvoet P: Decreased miR-181a expression in monocytes of obese patients is associated with the occurrence of metabolic syndrome and coronary artery disease. J Clin Endocrinol Metab 97: E1213-E1218, 2012.

8. Tam CS, Clément K, Baur LA and Tordjman J: Obesity and low-grade inflammation: A paediatric perspective. Obes Rev 11: $118-126,2010$

9. Geiss GK, Bumgarner RE, Birditt B, Dahl T, Dowidar N, Dunaway DL, Fell HP, Ferree S, George RD, Grogan T, et al: Direct multiplexed measurement of gene expression with color-coded probe pairs. Nat Biotechnol 26: 317-325, 2008.
10. Ouyang S, Li W, Liu Z, Li Y, Li S and Wu J: Association between serum soluble tumor necrosis factor- $\alpha$ receptors and early childhood obesity. Endocr J 63: 581-587, 2016.

11. Li H, Zong XN, Ji CY and Mi J: Body mass index cut-offs for overweight and obesity in Chinese children and adolescents aged 2-18 years. Zhonghua Liu Xing Bing Xue Za Zhi 31: 616-620, 2010 (In Chinese).

12. Vlachos IS, Paraskevopoulou MD, Karagkouni D, Georgakilas G, Vergoulis T, Kanellos I, Anastasopoulos IL, Maniou S, Karathanou K, Kalfakakou D, et al: DIANA-TarBase v7.0: Indexing more than half a million experimentally supported miRNA: MRNA interactions. Nucleic Acids Res 43 (Database Issue): D153-D159, 2015.

13. Franceschini A, Szklarczyk D, Frankild S, Kuhn M, Simonovic M, Roth A, Lin J, Minguez P, Bork P, von Mering C and Jensen LJ: STRING v9.1: Protein-protein interaction networks, with increased coverage and integration. Nucleic Acids Res 41 (Database Issue): D808-D815, 2013.

14. Smoot ME, Ono K, Ruscheinski J, Wang PL and Ideker T: Cytoscape 2.8: New features for data integration and network visualization. Bioinformatics 27: 431-432, 2011.

15. Huang da W, Sherman BT and Lempicki RA: Systematic and integrative analysis of large gene lists using DAVID bioinformatics resources. Nat Protoc 4: 44-57, 2009.

16. Ghanim H, Aljada A, Hofmeyer D, Syed T, Mohanty P and Dandona P: Circulating mononuclear cells in the obese are in a proinflammatory state. Circulation 110: 1564-1571, 2004.

17. Sayed D and Abdellatif M: MicroRNAs in development and disease. Physiol Rev 91: 827-887, 2011.

18. Lu LF and Liston A: MicroRNA in the immune system, microRNA as an immune system. Immunology 127: 291-298, 2009.

19. McGregor RA and Choi MS: MicroRNAs in the regulation of adipogenesis and obesity. Curr Mol Med 11: 304-316, 2011

20. Peng Y, Yu S, Li H, Xiang H, Peng J and Jiang S: MicroRNAs: Emerging roles in adipogenesis and obesity. Cell Signal 26: 1888-1896, 2014

21. Landgraf P, Rusu M, Sheridan R, Sewer A, Iovino N, Aravin A, Pfeffer S, Rice A, Kamphorst AO, Landthaler M, et al: A mammalian microRNA expression atlas based on small RNA library sequencing. Cell 129: 1401-1414, 2007.

22. Koh W, Sheng CT, Tan B, Lee QY, Kuznetsov V, Kiang LS and Tanavde V: Analysis of deep sequencing microRNA expression profile from human embryonic stem cells derived mesenchymal stem cells reveals possible role of let-7 microRNA family in downstream targeting of hepatic nuclear factor 4 alpha. BMC Genomics 11 (Suppl 1): S6, 2010.

23. Nesca V, Guay C, Jacovetti C, Menoud V, Peyot ML, Laybutt DR, Prentki $M$ and Regazzi R: Identification of particular groups of microRNAs that positively or negatively impact on beta cell function in obese models of type 2 diabetes. Diabetologia 56: 2203-2212, 2013.

24. Shi C, Zhang M, Tong M, Yang L, Pang L, Chen L, Xu G, Chi X, Hong Q, Ni Y, et al: MiR-148a is associated with obesity and modulates adipocyte differentiation of mesenchymal stem cells through Wnt signaling. Sci Rep 5: 9930, 2015.

25. Ortega FJ, Mercader JM, Catalán V, Moreno-Navarrete JM, Pueyo N, Sabater M, Gómez-Ambrosi J, Anglada R, Fernández-Formoso JA, Ricart W, et al: Targeting the circulating microRNA signature of obesity. Clin Chem 59: 781-792, 2013.

26. Jima DD, Zhang J, Jacobs C, Richards KL, Dunphy CH, Choi WW, Au WY, Srivastava G, Czader MB, Rizzieri DA, et al: Deep sequencing of the small RNA transcriptome of normal and malignant human $\mathrm{B}$ cells identifies hundreds of novel microRNAs. Blood 116: e118-e127, 2010.

27. Zhu $\mathrm{H}$ and Leung SW: Identification of microRNA biomarkers in type 2 diabetes: A meta-analysis of controlled profiling studies. Diabetologia 58: 900-911, 2015.

28. Wang J, Zhang Y, Zhang W, Jin Y and Dai J: Association of perfluorooctanoic acid with HDL cholesterol and circulating miR-26b and miR-199-3p in workers of a fluorochemical plant and nearby residents. Environ Sci Technol 46: 9274-9281, 2012.

29. Zhang Z, Kang Y, Zhang Z, Zhang H, Duan X, Liu J, Li X and Liao W: Expression of microRNAs during chondrogenesis of human adipose-derived stem cells. Osteoarthritis Cartilage 20: 1638-1646, 2012

30. Wojcicka A, Swierniak M, Kornasiewicz O, Gierlikowski W, Maciag M, Kolanowska M, Kotlarek M, Gornicka B, Koperski L, Niewinski G, et al: Next generation sequencing reveals microRNA isoforms in liver cirrhosis and hepatocellular carcinoma. Int J Biochem Cell Biol 53: 208-217, 2014. 
31. Fornari F, Milazzo M, Chieco P, Negrini M, Calin GA, Grazi GL, Pollutri D, Croce CM, Bolondi L and Gramantieri L: MiR-199a-3p regulates mTOR and c-Met to influence the doxorubicin sensitivity of human hepatocarcinoma cells. Cancer Res 70: 5184-5193, 2010.

32. Yin J, Hou P, Wu Z, Wang T and Nie Y: Circulating miR-375 and miR-199a-3p as potential biomarkers for the diagnosis of hepatocellular carcinoma. Tumour Biol 36: 4501-4507, 2015.

33. Ji H, Chen M, Greening DW, He W, Rai A, Zhang W and Simpson RJ: Deep sequencing of RNA from three different extracellular vesicle (EV) subtypes released from the human LIM1863 colon cancer cell line uncovers distinct miRNA-enrichment signatures. PLoS One 9: e110314, 2014.

34. Weisberg SP, McCann D, Desai M, Rosenbaum M, Leibel RL and Ferrante AW Jr: Obesity is associated with macrophage accumulation in adipose tissue. J Clin Invest 112: 1796-1808, 2003.
35. He $X$ and Zhang $\mathrm{J}$ : Why do hubs tend to be essential in protein networks? PLoS Genet 2: e88, 2006

36. Jeong H, Mason SP, Barabási AL and Oltvai ZN: Lethality and centrality in protein networks. Nature 411: 41-42, 2001.

37. Tabur S, Oztuzcu S, Oguz E, Korkmaz H, Eroglu S, Ozkaya M and Demiryürek AT: Association of Rho/Rho-kinase gene polymorphisms and expressions with obesity-related metabolic syndrome. Eur Rev Med Pharmacol Sci 19: 1680-1688, 2015.

38. Huang N, Wang J, Xie W, Lyu Q, Wu J, He J, Qiu W, Xu N and Zhang Y: MiR-378a-3p enhances adipogenesis by targeting mitogen-activated protein kinase 1 . Biochem Biophys Res Commun 457: 37-42, 2015.

39. Qiu J, Cheng R, Zhou XY, Zhu JG, Zhu C, Qin DN, Kou CZ and Guo XR: Gene expression profiles of adipose tissue of high-fat diet-induced obese rats by cDNA microarrays. Mol Biol Rep 37: 3691-3695, 2010 